\title{
Visual imagery differences and eye movements in the recall of pictures*
}

\author{
DAVID F. MARKS \\ Lniversity of Otago, Dunedin, New Zealand
}

\begin{abstract}
Groups of vivid and poor visualizers were given a picture memory task. and horizontal and vertical components of the electro-oculogram were recorded. This allowed a detailed investigation of each S's eve movements in the perception, imagery, and recall phases of the task. The vivid visualizers gave a higher accuracy of recall. Eye movement rate was lower in visual imagery than it was in perception, especially in the group of vivid visualizers. There was some evidence of scanning activity prior to recall, but only if positional cues were provided or if recall was incorrect. No scanning occurred prior to accurate recall unprompted by a positional cue. These results provide no support to the theories of image construction proposed by Hebb $(1949,1968)$ and Neisser $(1967)$. As suggested by Singer $(1966)$, an absence of eye movement may be a necessary condition for vivid visual imagery.
\end{abstract}

Do the eyes "scan" a visual image in much the same way as they glance at physical stimuli in perception? Hebb $(1949,1968)$ has proposed that eye movements have an essential organizing function in visual imagery, as they have in visual perception. In Hebb's (1968) view, "If the image is a reinstatement of the perceptual process it should include the eye movements (and in fact usually does) [p. 470]." In similar vein, Neisser (1967) suggested that imagery vividness is a continuum "loosely correlated with the extent to which scanning eye movements are involved... Visual synthesis of an image without eye motion may be possible, but the better the image the more likely it is to involve some sort of scanning [p. 153]." That the processes of perception and imagery are continuous is clearly a theoretically attractive notion, and there is evidence that images and percepts in the same modality may share the same central processing mechanisms (Brooks, 1968; Atwood. 1971: Segal, 1971).

At the peripheral level, evidence on imagery perception continuity is, at best, equivocal. A number of investigators of dream imagery (e.g., Dement \& Kleitman, 1957; Dement \& Wolpert, 1958: Roffwarg, Dement, Muzio, \& Fisher, 1962) have concluded that rapid eye movements (REMs) observed during sleep result from the "scanning" of dream episodes. More recent evidence (Oswald, 1970; Jacobs, Feldman, \& Bender, 1972: Koulack, 1972) casts doubt on the "looking-at-dream-pictures" theory since, with sensitive recording procedures, REM periods may be observed in the sleep patterns of congenitally-blind Ss (Amadeo \& Gomez, 1966: Gross, Byrne, \& Fisher, 1965). Other

\footnotetext{
*The author would like to thank Ulric Neisser for his helpful comments on an earlier version of this article, although full responsibility for the conclusions lies with the author. The author also thanks Peter McKellar for providing research facilities and Beverley Barron, Terry Henry, and Pamela Ruddenklau for their assistance in the collection and analysis of data. Reprints may be obtained from David Marks, Department of Psychology, University of Otago, Box 56, Dunedin, New Zealand.
}

facts which are difficult to explain are the prominence of REMs in sleep of neonates (Roffwarg, Dement, \& Fisher, 1964; Parmelee, Wenner, Akiyama, Schultz, \& Stern, 1964), of kittens reared in total darkness (Fishbein, Schaumberg, \& Weitzmann, 1965), of monkeys reared without patterned visual experience (Berger \& Meier, 1965), and in the decorticate human and animal (Jouvet, 1970). In no study has a perfect one-to-one relationship been observed between REM periods and dreaming (Dement, 1965), and in in one small-scale study conducted in our own laboratory (Marks, Hurst, Pringle, \& Ruddenklau, 1972), the proportions of dream reports following 20 REM and 20 non-REM awakenings were actually identical-40\% exactly. Jacobs, Feldman, and Bender (1972) recorded dc electro-oculograms (EOGs) and observed a matching between eye movements and dream activity for only $18 \%$ of 50 dream reports. While dream reports were highly variable, eye movement patterns during REM periods were consistent both within and across Ss and showed no resemblance to eye movement patterns of the awake state.

The case for a positive correlation between image vividness and eye movements in waking imagery is no stronger. Hale and Simpson (1970) obtained vividness ratings under three conditions in which the Ss were encouraged to move their eyes, the eye movements were imagined, and the eyes were kept still. No difference in vividness occurred between these conditions. Brown (1968) compared the ocular activity of vivid and poor visualizers during imagined pursuit and found no consistent differences between groups. Zikmund (1972) found that only in about $17 \%$ of Ss were there consistent nystagmoid eye movements accompanying vivid imagery of movement.

The purpose of the following experiment was to test for image scanning with groups of vivid and poor visualizers in a picture-recall task. If Hebb's (1949. 1968) formulation is correct, then, assuming no $S$ to be totally devoid of visual imagery. scanning motions 
should accompany picture recall in both groups of Ss. On Neisser's (1967) theory, in which scanning is positively correlated with vividness although not essential, relatively more scanning behavior should be observed in the group of vivid visualizers. ${ }^{1}$

\section{Subjects}

The Ss were 16 volunteers aged 16-18 years selected from a total of 116 young people who had completed the vividness of visual imagery questionnaire (VVIQ). All Ss were from top streams of local secondary schools and satisfied New Zealand university entrance requirements. The VVIQ is a brief 16 -item questionnaire in which the image summoned for each item is rated along a 5-point scale of vividness, once with the eyes open, and once with the eyes closed (for further details, see Marks, 1973). On the basis of total scores on the VVIQ, eight of the lowest scorers (mean rating $=1.53$ ) and eight of the highest scorers (mean rating $=3.15$ ) were selected to form two experimental groups, designated "vivid" and "poor visualizers," respectively. There were four males and four females in each group.

\section{Stimuli}

The stimuli were 10 colored photographs reproduced as transparencies. Five of these were photographs of sets of 15 unrelated objects in a random arrangement (for examples, see Marks, 1972, 1973) and five displayed photographs of complete scenes (a Venice canal, a Turkish marketplace, a street scene, a stage coach, and a group of bathers). Two further slides (Eiffel Tower, and a street scene) were used during two practice trials.

\section{Apparatus}

Eye movements were recorded by means of Beckman Biopotential Skin Electrodes. The horizontal component of the EOG was obtained from two electrodes attached $1 \mathrm{~cm}$ away from the outer canthi of the eyes, and the vertical component of the EOG from two electrodes placed $1, \mathrm{~cm}$ directly above and below the right eye. The two EOG components were amplified and recorded with dc settings on a Beckman Type RB Dynograph. S sat in a dimly lighted $(0.010-0.016 \mathrm{fL})$, electrically-insulated room with his head in a headrest to minimize head movement artifacts. The stimulus display area was $93 \times 65 \mathrm{~cm}$ at a distance of $1 \mathrm{~m}$ in front of S's eye, giving a stimulus area of $50 \times 36 \mathrm{deg}$. Stimuli were presented using a $35 \mathrm{~mm}$ slide projector in an adjoining room from a position above and behind S's head. An intercom provided a communication link with $S$ during the experiment.

\section{Procedure}

After electrodes were attached, $S$ was seated with his head in the headrest and asked to keep his eyes open throughout the experiment. A calibration chart was presented, and $S$ fixated the center and other points of this display to allow adjustment of the EOG amplifiers and calibration of the two EOG components. Sensitivity for different Ss varied between $0.5 \mathrm{~mm}$ and $2.5 \mathrm{~mm}$ deflection per degree of eye movement. Ss were told that the electrodes were for recording "brain waves," and through questioning at the end of the experiment, they were all found to be unaware that eye movements had been recorded.

Each trial consisted of four stages: stimulus presentation $(20 \mathrm{sec})$, a delay $(40 \mathrm{sec})$, a 5 -point vividness rating $(10 \mathrm{sec})$, and questioning $(75 \mathrm{sec})$. Five multiple-choice questions were asked orally on each trial at the rate of one per $15 \mathrm{sec}$ by an $E$ who did not know which group a given $S$ was in. $A$ second $E$ monitored the EOG recorder, and there was no obvious way in which the manner of questioning (e.g., tone of voice, stressing, pause allocation, etc.) could be dependent on the EOG record. S selected his response from the three answers provided. ${ }^{2}$ The delay between stimulus presentation and the vividness rating allowed afterimages to disappear, and during the first $30 \mathrm{sec}$ of this delay, $\mathrm{S}$ was required to perform a serial subtraction task. This provided a method for minimizing verbal rehearsal (Peterson \& Peterson, 1959). Ss were told: "After each slide has been presented for a short period of time, a three-figure number will be read to you. As soon as you hear this number, count back from it in threes until you hear the word "Stop." For example, if you hear "7-9-4," start counting "7-9-4, 7-9-1, 7-8-8," etc. Count aloud and count as quickly as you can. During this counting period, you should try to keep in mind a picture of the displayed slide. This should include, as far as possible, details of the shape, color, and relative positions of different aspects of the picture displayed." Prior to the ten experimental trials, each $S$ was given two practice trials, one to practice subtraction, imaging, and rating, and the second to practice the complete procedure. Drift of the eye position recordings was corrected before each trial. The records obtained from two $S$ s contained too much drift to allow a reliable analysis, and so these $S$ s were replaced. The various stages of each trial, including the start and finish of questions and the onset of S's responses were marked on the polygraph chart paper using an event recorder. After the experimental trials had been completed, the two EOG components were again calibrated.

\section{RESULTS}

\section{Accuracy of Recall}

The mean numbers of correct responses obtained from the groups of vivid and poor visualizers were 35.7 and 30.5 , respectively. In a 3-way ANOVA, this difference was highly significant $[F(1,12)=14.62$, $\mathrm{p}<.005]$. The superior recall of the vivid imagers provided an objective validation of the VVIQ used to select the Ss and to divide them into the two experimental groups. Females gave more accurate recall than males $[\mathrm{F}(1,12)=8.49, \mathrm{p}<.025]$ but the accuracy of recall for the scenes and the montages was exactly equal. None of the interactions was significant. No consistent difference was observed in the counting rates of the vivid and poor visualizers, and it is, therefore, unlikely that the superior accuracy of the vivid imagers was due to more rehearsal by these Ss.

\section{Vividness}

The mean vividness ratings obtained from the vivid and poor visualizers were 2.68 and 3.26 , respectively $[F(1,12)=6.75, p<.025]$. As expected, the vivid visualizers, selected for their reports of more vivid imagery using the VVIQ, reported the more vivid imagery in the experimental task. It is noteworthy that both groups of Ss, although especially the vivid visualizers, reported their experimental imagery to be lower in vividness than the imagery they obtained when completing the VVIQ prior to the experiment (cf. Sheehan \& Neisser, 1969). The scenes received ratings of greater vividness than the montages $[F(1,12)=19.52$, 
$p<.001]$. and this effect occurred for both groups of Ss.

\section{Accuracy Related to Vividness}

As indicated above, between groups of Ss selected for their reports of low or high vividness, there was a highly significant difference in accuracy. As indexed by the correlation coefficient, the relationship across Ss between accuracy and vividness was higher for the montages $(r=0.59$ than for scenes $(r=0.34)$. In contrast to the between-groups effect, there was virtually no intra-S correlation between accuracy and vividness. The rating on the trial with the fewest errors was compared to that obtained on the trial with most errors. The mean rating for best recall of $2.79 \mathrm{did}$ not differ significantly from the mean of 2.70 obtained for worst recall $(t<1.0)$. These aspects of the results are reported in more detail elsewhere (Marks, 1972, 1973).

\section{Eye Movement Rate}

For each $S$, the eye movement rate (EMR) was calculated for the viewing and imaging phases of the task. For this analysis, the horizontal component of the EOG was divided into 1-sec intervals and the amplitude of any change in this component over each interval was determined. Mean EMRs in degrees per second of vivid and poor visualizers for perception and imagery are shown in Table 1. No differences occurred in EMR between the scenes and the montages, and so this factor was dropped.

The 2 by 2 ANOVA showed that EMR was significantly lower in imagery than in perception $[F(1,14)=8.73, p<.025]$. Both the vividness effect and the interaction failed to reach statistical significance $[F(1,14)=2.96$, and 2.72 , respectively $]$, but a simple test of vividness on EMR in the imagery condition indicated a significant effect $[F(1,14)=5.72, p<.05]$. Hence, Ss who reported more vivid visual imagery and who produced more accurate recall showed a lower rate of eye movement during visual imagery prior to recall. This result is the exact opposite to that predicted by Neisser (1967).

\section{Eye Movement Path}

This analysis investigated the two EOG components for evidence of "image scanning." Segments of the EOG records which corresponded to a subset of 20 questions involving the periphery of a stimulus display were analyzed. The visual field was divided into a 3 by 3 matrix of areas in which the central area was a $4 \times 4 \mathrm{deg}$ square. By examining the pen record of the two orthogonal EOG components, it was possible to construct each S's eye movement path (EMP), i.e., at each point of time, to determine where S's eyes were looking. Clearly, this determination was relative to each
Table 1

Mean EMRs of Vivid and Poor Visualizers Associated With Perception and Imagery in Degrees Per Second

\begin{tabular}{lccc}
\hline Group & Perception & Imagery & Means \\
\hline Vivid Visualizers & 6.81 & 3.62 & 5.21 \\
Poor Visualizers & 7.13 & 6.22 & 6.67 \\
Means & 6.97 & 4.92 & 5.94 \\
\hline
\end{tabular}

S's individual EOG calibration. Where a blink occurred in a relevant part of the record of the vertical EOG component, a straight line was drawn between the onset and the offset of the blink. In the majority of the cases, blinks were readily distinguishable from actual eye movements by their jagged contour. One S's blinks were too hard to identify reliably, and so he was replaced.

Ten of the questions selected for this analysis explicitly referred to a position (e.g., "How many balconies did the house on the extreme right have: one, two, or three"?) and ten questions provided no explicit positional cue (e.g., "What color was the goose's beak: yellow. red, or black"?). Each of the 20 EOG segments started halfway through a question and ended $0.50 \mathrm{sec}$ before S's response. Their mean duration was $4.59 \mathrm{sec}$. For each segment, the percentage of the EMP not in the central cell was calculated. To partial out the effect of accuracy, this analysis was done separately for EMP segments prior to correct and incorrect recall. Mean values for the two groups of Ss are given in Table 2. No differences occurred in EMP measures between the scenes and the montages, and so in this, and the next, analysis, this factor was dropped.

Although there was a greater tendency among the vivid visualizers to stare at the center of the field, error variance was high and a 2 by 2 by 2 ANOVA gave F less than one for this effect. ${ }^{3}$ An important result was the greater tendency for the eyes to wander from the center of the field prior to recall which was incorrect $[F(1,14)=4.82, \quad p<.05]$. When a position was explicitly mentioned in the question, the eyes also moved off center for a greater proportion of the recall period $[F(1,14=3.33, \mathrm{p}<.10]$. For all interactions, $F$ was less than one.

In a final, and more crucial, analysis, the EMP data were investigated in more detail for evidence of "image scanning." For each recall episode, the percentage of noncentral EMP on the appropriate reference area was calculated. With eight peripheral areas, and only one of them appropriate, then, provided these areas were equal in size and that Ss showed no response bias (e.g.. to look more towards the right), the mean rate of response would be $12.5 \%$. However, neither of these conditions held and hence a more appropriate chance level was calculated. Data on the average percentage looking time in the eight peripheral areas gave an empirically derived chance level of $11.6 \%$ for both groups of Ss. ${ }^{4}$ If Ss "scanned" their visual images, then mean percentage EMP on an appropriate reference area would be significantly greater than this value. Mean scores of the 
Table 2

Mean Percentages of Noncentral EMP in Correct and Incorrect Recall of Vivid and Poor Visualizers When Positional Cues Were Explicit and When They Were Not

\begin{tabular}{|c|c|c|c|c|c|}
\hline Group & \multicolumn{2}{|c|}{ Correct Recall } & \multicolumn{2}{|c|}{ Incorrect Recall } & Means \\
\hline
\end{tabular}

vivid and poor visualizers for the two types of question are presented in Table 3.

A 2 by 2 by 2 ANOVA gave a nonsignificant between-groups effect $(F<1.0)$ but a significant effect for type of question $[F(1,14)=5.09, p<.05]$. For questions containing an explicit positional cue, the mean score was significantly greater than the chance level ( $p<.01$ for correct recall $; \mathrm{p}<.05$ for incorrect recall). For questions not providing an explicit positional cue, however, the mean values of $9.19 \%$ for correct recall and $20.19 \%$ for incorrect recall did not differ statistically from the chance level. Of signal importance was the finding that the eyes travelled over the reference area prior to incorrect recall for a greater proportion of time than they did prior to correct recall $[F(1.14)=3.93$, $p<.10]$. All other $F$ values were less than one. Error variance was again high, and much of this was contributed by the mean EMP scores for incorrect recall which were based on a relatively small sample of each S's ocular activity. An ANOVA conducted on scores for correct recall only brought out the explicitness effect at the $1 \%$ level $[F(1,14)=11.30]$.

The mean accuracy of recall for questions which explicitly directed $S$ to a part of the picture was 6.94 as compared to 6.56 for nonexplicit questions $(t=0.77)$. It could be argued that the lower accuracy obtained with nonexplicit questions is evidence that $S s$ did not remember in what part of the picture the critical item was located, thereby accounting for the lower EMP scores for these questions. While it seems unlikely that such a small difference in accuracy could cause such a large difference in eye movements, this hypothesis is still less plausible in the light of the fact that the Ss spent more time looking at the reference area prior to recall which was totally incorrect. One clear result was that correct recall episodes uncontaminated by positional cues provided no support for the "image-scanning" hypotheses proposed by Hebb and Neisser.

\section{DISCUSSION}

The results obtained suggest a number of conclusions. First, none of the measures of ocular activity employed in this study allow any definite support for the "image-scanning" hypothesis proposed by Neisser (1967). There was no evidence that vivid visualizers showed more scanning activity than a group of Ss operationally defined to be poor at visualizing. On the contrary, for one measure (EMR), reported vividness of visual imagery and eye-movement activity were negatively correlated. These results therefore support those of many other investigators of both waking and dream imagery who have found no consistent evidence of a positive relationship between vividness and scanning behavior (for references, see the Introduction).

Second, in correct recall, when positional or directional cues were not provided, there was no evidence of any type of scanning in either vivid or poor visualizers. This result, therefore, lends no support to the theory of Hebb $(1949,1968)$ in which eye movements are supposed to be necessary for image construction. This result has no bearing on Hebb's (1968) second theory of imagery which states that it is necessary only to imagine the eye movements. As stated, the latter theory seems difficult to test.

Third, in the case of incorrect recall, there was evidence of scanning, although probably not "image scanning." It seems unlikely that images are not scanned if they are well-formed and accurate but are scanned if they are incomplete and inaccurate. One interpretation

Table 3

Mean Percentages of Noncentral EMP on the Reference Area in Correct and Incorrect Recall of Vivid and Poor Visualizers When Positional Cues Were Explicit and When They Were Not

\begin{tabular}{|c|c|c|c|c|c|}
\hline Group & \multicolumn{2}{|c|}{ Correct Recall } & \multicolumn{2}{|c|}{ Incorrect Recall } & Means \\
\hline
\end{tabular}

$t_{p}<.01 \quad * p<.05$ 
of this novel finding is that the eye movements were scanning, not images, but the environment, for cues to aid recall. The powerful role of spatial layout in mnemonic systems is well documented (Yates, 1966) and confirmed by a number of experimental studies (e.g., Ross \& Lawrence, 1968; Crovitz, 1969; Briggs, Hawkins, \& Crovitz, 1970; McKellar, Marks, \& Barron, 1973). These studies make it clear that if there is a visual association between some information and a particular location then the recall of the location provides a strong cue for the recall of the desired information. In this experiment, in which the Ss' eyes were open, scanning the location of some critical item, where the location was remembered but not the item, could have played a similar role.

Fourth, some sort of scanning took place when recall was explicity directed toward the location of the required information on the stimulus display. As in the case of the scanning motions observed prior to incorrect recall, it is more likely to have been the environment, and not the image, that was being scanned. Any tendency to look for cues in the location of the information to be recalled is likely to be stronger when this location is specifically referred to in the E's request for that information. The absence of any scanning activity prior to correct recall, when positional cues were not explicitly provided, would seem to make the "image-scanning" hypothesis an unnecessary one. Only further experimentation, in which recall occurs with closed eyes, can test these speculations.

While the present data offer no support to the image-scanning notion, one should perhaps be careful not to overgeneralize to classes of imagery not included within the present study. Antrobus, Antrobus, and Singer (1964) demonstrated that active visual imagery produced more eye movements than static imagery. Also of relevance here is research in which optokinetic nystagmus has been used as an objective criterion for hypnotically induced visual hallucinations (Brady \& Levitt, 1964, 1966). In these studies, the stimulus used to elicit visual imagery was a rotating drum covered with black and white vertical stripes. On the negative side, Graham's (1970) results indicate that movements in imagery differ from those occurring in perception. While evidence of a vividness-scanning relationship was lacking in her data, Brown (1968) did observe eye motions during imagery similar in general appearance to those which occurred during perception of the stimulus. Again, this was a moving stimulus, a beating metronome. Lenox, Lange, and Graham (1971), however, found that when the eyes were closed the amplitude of the imagery eye motions actually exceeded that of the movements during perception. Zikmund (1972) observed, under some conditions, a positive correlation between nystagmus and vivid visual imagery. Clearly then, there is evidence of eye motion during imagery of movement, and the relationship between image vividness and ocular activity may differ entirely for such imagery. Whether the eye movements accompanying active imagery are actually "scanning" motions, have some other function such as image generation, or are part of a motor recall process is a debatable and researchable question. In the case of eidetic imagery, scanning motions of the eyes have been used as a defining criterion (Haber \& Haber, 1964). Hypnopompic imagery, hypnagogic imagery, and spontaneous visual hallucinations all constitute other classes of imagery experience which may or may not include scanning responses. In sum, the present negative results may relate solely to the recall of pictures that are not moving.

One may inquire at this point if there appear to be any critical conditions under which scanning of static images may be expected to occur. What were the crucial factors in this experiment that caused vividness and ocular activity to show either no relationship or, as was true of EMR, a reciprocal one? Singer (1966) and Singer, Greenberg, and Antrobus (1971) suggested that images and percepts are two classes of stimuli competing for space in the same central processing channel. Depending on the state of the $S$ and the external environment that surrounds him, $S$ may focus attention to a varying degree upon either class of stimuli: internal (includes images) or external (percepts). In Singer's model there is a reciprocal relationship between the rates of processing of external and internal stimuli. We can extend this model by the suggestion that vividness of imagery and vividness of perception may also, to some extent, be reciprocally related. Any condition, whether $S$ related (e.g., eyes still, eyes closed, or hypnotic effects) or environment related (e.g., poor illumination or monotonous stimulation), that involves a reduction or degradation of novel sensory input will, therefore, be conducive to the occurrence of visual imagery that is relatively vivid. A truly vivid image of hallucinatory quality is normally a rare phenomenon, difficult to obtain in most fully awake adult Ss. Where a vivid image is needed and some external stimulation is present, as in our picture memory task, the fewer the number of eye movements, the better the image is likely to be. Contrary to the view of Neisser (1967) and Hebb (1968), therefore, an absence of eye movement may sometimes be a necessary condition for image construction. Given ideal conditions for the occurrence of a vivid image, as in the case of an $S$ who is an eidetiker, or hallucinating, as a result of hypnotic suggestion, schizophrenia, or the administration of a hallucinogen, accompanying eye motions may be expected. Even in these cases, however, there is the possibility that the eye movements themselves may be imagined, as Hebb (1968) has suggested.

\section{REFERENCES}

Amadeo. M., \& Gomez. E. Eye movements. attention. and dreaming in subjects with lifelong blindness. Canadian Psychiatric Association Journal, 1966, 11. 500-507. 
Antrobus, J. S.. Antrobus, J. S., \& Singer, J. L. Eye movements accompanying daydreaming, visual imagery, and thought suppression. Journal of Abnormal \& Social Psychology, 1964, 69, 244-252.

Atwood. G. An experimental study of visual imagination and memory. Cognitive Psychology, 1971, 2, 290-299.

Berger, R. J., \& Meier, G. W. Eye movement during sleep and waking in infant monkeys (Macaca mulatta) deprived of pattern vision. Developmental Psychobiology, 1965, 12, 257-277.

Brady, J. P., \& Levitt, E. E. Nystagmus as a criterion of hypnotically induced visual hallucinations. Science, 1964, $146,85-86$

Brady, J. P., \& Levith, N. E. Hypnotically induced visual hallucinations. Psychosomatic Medicine, 1966, 28, 351-363.

Briggs, G. G., Hawkins, S., \& Crovitz, H. F. Bizarre images in artificial memory. Psychonomic Science, 1970, 19, 353-354.

Brooks, L. R. Spatial and verbal components of the act of recall. Canadian Joumal of Psychology, 1968, 22, 349-368.

Brown, B. B. Visual recall ability and eye movements. Psychophysiology, 1968, 4, 300-306.

Crovitz, H. F. Memory loci in artificial memory. Psychonomic Science, 1969, 19, 82-83.

Dement, W. C. An essay on dreams: The role of physiology in understanding their nature. New Directions in Psychology, $1965,2,135-257$

Dement, W. C., \& Kleitman, N. The relation of eye movements during sleep to dream activity: An objective method for the study of dreaming. Journal of Experimental Psychology, $1957,53,339-346$.

Dement, W. C., \& Wolpert, E. A. The relation of eye movements; body motility and external stimuli in dream content. Journa of Experimental Psychology, 1958, 55, 543-553.

Fishbein, W., Schaumberg, H., \& Weitzmann, E. D. Rapid eye movements during sleep in dark reared kittens. Paper presented at the annual meeting of the Association for the Psychophysiological Study of Sleep, Washington, D.C., March 1965.

Gould, J. D., \& Schaffer, A. Eye movement patterns in scanning numeric displays. Perceptual \& Motor Skills, 1965, 20 , $521-535$

Graham, K. R. Optokinetic nystagmus as a criterion of visual imagery. Journal of Nervous \& Mental Disease, 1970, 151, $411-414$.

Gross, J., Byrne, J., \& Fisher, C. Eye movements during emergent Stage I EEG in subjects with life-long blindness. Journal of Nervous \& Mental Disease, 1965, 141, 365-370.

Hale, S. M., \& Simpson, H. M. Effects of eye movements on the rate of discovery and the vividness of visual images. Perception \& Psychophysics, 1970, 9, 242-246.

Hebb, D. O. Organization of behavior. New York: Wiley, 1949.

Hebb, D. O. Concerning imagery. Psychological Review, 1968 , $75,466-477$.

Jacobs, L., Feldman, M., \& Bender, M. B. Are the eye movements of dreaming sleep related to the visual images of the dreams? Psychophysiology, 1972, 9, 393-401.

Jouvet, M. Neurophysiology of the states of sleep. In G. C. Quarton, T. Milnechuk, and F. O. Schmitt (Eds.), The neurosciences: A study program. New York: Rockefeller University Press, 1970. Pp. 529-544.

Koulack, D. Rapid eye movements and visual imagery during sleep. Psy chological Bulletin, 1972, 78, 155-158.

Lenox, J. R., Lange, A. F., \& Graham, K. R. Eye movement amplitudes in imagined pursuit of a pendulum with the eyes closed. Psychophysiology, 1970, 6, 773-777.

McKellar, P., Marks, D. F., \& Barron, B.F. The mnemonic walk and visual imagery differences. In preparation, 1973.

Marks, D. F. Individual differences in the vividness of visual imagery and their effect on function. In P. W. Sheehan (Ed.), The function and nature of imagery. New York: Academic Press, 1972. Pp. 83-108.

Marks, D. F. Visual imagery differences in the recall of pictures.
British Journal of Psychology, 1973, 64, 17-24.

Marks, D. F., Hurst, J., Pringle, J., \& Ruddenklau, P. Unpublished experiment, 1972.

Neisser, U. Cognitive psychology. New York Appleton-Century-Crofts, 1967.

Neisser, U. Changing conceptions of imagery. In P. W. Sheehan (Ed.), The function and nature of imagery. 1972.

Oswald, I. Sleep. (2nd Ed.) Harmondsworth: Penguin, 1970.

Parmelee, A. H., Jr., Wenner, W. H., A kiyama, Y., Schultz, M., \& Stern, E. Sleep states in premature infants. Developmental Medicine \& Child Neurology, 1967, 9, 70-77.

Peterson, L. R., \& Peterson, M. J. Short-term retention of individual verbal items. Journal of Experimental Psychology, $1959,58,193-198$.

Roffwarg, H. P., Dement, W. C., Muzio, J. N., \& Fisher, C. Dream imagery: Relation to rapid eye movements of sleep. Archives of General Psychiatry, 1962, 7, 235-258.

Roffwarg, H. P., Dement, W. C., \& Fisher, C. Observations on the sleep-dream pattern in neonates, infants, children, and adults. In E. Harms (Ed.), Problems of sleep and dreams in children London: Pergamon, 1964.

Ross, J., \& Lawrence, K. A. Some observations on memory artifice. Psychonomic Science, 1968, 13, 107-108.

Segal, S. J. Processing of the stimulus in imagery and perception. In S. J. Segal (Ed.), Imagery: Current cognitive approaches. New York and London: Academic Press, 1971. Pp. 69-100.

Sheehan, P. W., \& Neisser, U. Some variables affecting the vividness of imagery in recall. British Journal of Psychology, $1969,60,71-80$.

Singer, J. L. Daydreaming: An introduction to the experimental study of inner experience. New York: Random House, 1966.

Singer, J. L., Greenberg, S., \& Antrobus, J. S. Looking with the mind's eye: Experimental studies of ocular motility during daydreaming and mental arithmetic. Transactions of the New York A cademy of Sciences, 1971, 33, 694-709.

Yates, F. A. The art of memory. London: Routledge \& Kagan Paul, 1966.

Zikmund, V. Physiological correlates of visual imagery. In P. W. Sheehan (Ed.), The function and nature of imagery. New York: Academic Press, 1972. Pp. 355-387.

\section{NOTES}

1. Dr. Neisser's more recent conception of imagery may be found in Neisser (1972).

2. Further details on the questions may be obtained upon request from the author.

3. In a similar experiment to this one (unpublished), vivid visualizers looked toward the $4 \times 4 \mathrm{deg}$ central area for an average of $26.4 \%$ of the total recall time, as compared to $13.6 \%$ in the group of poor visualizers. This difference was highly significant $(p<.005)$. This effect should be interpreted cautiously because $i^{*}$ is confounded with another result in which the effective visual field during imagery is smaller in area for vivid visualizers than for poor visualizers. In the present study, the imagery eye movements of vivid visualizers were contained within a mean area of $19 \times 14 \mathrm{deg}$, as compared to $26 \times 18 \mathrm{deg}$ in the case of poor visualizers. Hence, the $4 \times 4 \mathrm{deg}$ central area occupied $6.0 \%$ and $3.2 \%$ of the total effective fields of the vivid and poor visualizers, respectively.

4. The stated chance level was derived from data on the percentages of noncentral looking time spent in each of the peripheral areas. The two groups of Ss showed no consistent differences, so the data were pooled. Starting in the top right-hand corner and working clockwise, these percentages were $9.8,13.4,12.9,24.2,5.7,12.8,5.3$, and 15.9 (cf. Gould \& Schaffer, 1965). There were marked biases toward the right-hand and bottom sides. The distribution of reference points over these areas was $5,1,2,3,3,3,3,0$. This gave a mean chance score of $11.61 \%$.

(Received for publication July 10, 1972; revision received April 19, 1973.) 